\title{
HYPERBOLICITY OF LINEAR PARTIAL DIFFERENTIAL EQUATIONS WITH DELAY *
}

\author{
ANDRÁS BÁTKAI ${ }^{\dagger}$
}

\begin{abstract}
Robust hyperbolicity and stability results for linear partial differential equations with delay will be given and, as an application, the effect of small delays to the asymptotic properties of feedback systems will be analyzed.
\end{abstract}

\section{Introduction}

Partial differential equations with delay have been studied for many years and by many different methods. In an abstract way and using the standard notation (see [33]), they can be written as

$$
\left\{\begin{array}{l}
u^{\prime}(t)=B u(t)+\Phi u_{t}, \quad t \geq 0, \\
u(0)=x, \\
u_{0}=f,
\end{array}\right.
$$

in a Banach space $X$, where $(B, D(B))$ is a (unbounded) linear operator on $X$ and the delay operator $\Phi$ is supposed to belong to, e.g., $\mathcal{L}\left(W^{1, p}([-1,0], X), X\right)$ for some $1 \leq$ $p \leq \infty$. J. Hale [13], G. Webb [32], N. Krasovski and others were the first to apply semigroup theory to delay equations, and we refer to [33] for more recent references on partial differential equations with delay.

As a first step one has to choose an appropriate state space. One of the possibilities is to work in the space of continuous $X$-valued functions. In this case, the relationship between solutions of (DE) and a corresponding semigroup has been studied intensively (see for example [14], 33] or [8, Section VI.6]) and is well understood. On the other hand, the state space $\mathcal{E}:=X \times L^{p}([-1,0], X)$ turns out to be a very good choice with regards to certain applications (e.g., to control theory, see [26], to numerical methods, see [19]), because we can use the reflexivity or the Hilbert space structure of the state space. This approach will be used in this paper.

*TO APPEAR IN INTEGRAL EQUATIONS AND OPERATOR THEORY

${ }^{\dagger}$ The author thanks W. Desch (Graz), I. Győri (Veszprém) and R. Schnaubelt (Halle) for helpful discussions. 
The aim of this work is to give robust hyperbolicity and stability results for linear partial differential equations with delay, especially for the cases where no spectral mapping theorems are available and we cannot use the powerful technics of characteristic equations. As an application, we analyze the effect of small delays to the asymptotic properties of feedback systems.

In the next section we collect some results on the semigroup approach for delay equations in the $L^{p}$ history space, mainly from [1. This approach is especially useful in the Hilbert space case because the theorem of Gearhart is available and allows stability results in the case where the semigroup generated by $(B, D(B))$ is not compact, see [2] for applications.

In Section 3, we present robust hyperbolicity results in the Hilbert space context of the following kind. Assume, that $(B, D(B))$ generates a hyperbolic semigroup and that the delay operator $\Phi$ is "small" in some sense, which will be explaind in the text later on. Then the delay semigroup remains hyperbolic. As a special case we consider uniform exponential stability.

In the last section we investigate the important question of the effect of small delays. The problem is the following: We consider delays of the special form $\Phi:=C \delta_{-\tau}$. The question is, knowing that the solutions of the system are exponentially stable for $\tau=0$, whether it follows that they remain stable for arbitrary small $\tau>0$. This question is motivated by feedback-systems and control theory and we give more references on this question in the text. First two examples are given to show how the stability can be destroyed and then a general approach to treat this question is provided. The problem is considered for norm continuous semigroups and for commuting compact perturbations.

\section{The semigroup approach to delay equations}

Let us summarize here some results from [1] on the semigroup approach to linear partial differential equations with delay.

Consider the equation

$$
(\mathrm{DE}) \quad\left\{\begin{array}{l}
u^{\prime}(t)=B u(t)+\Phi u_{t}, \quad t \geq 0, \\
u(0)=x, \\
u_{0}=f,
\end{array}\right.
$$

where

- $x \in X, X$ is a Banach space,

- $B: D(B) \subseteq X \longrightarrow X$ is a linear, closed, and densely defined operator,

- $f \in L^{p}([-1,0], X), p \geq 1$,

- $\Phi: W^{1, p}([-1,0], X) \longrightarrow X$ is a linear, bounded operator,

- $u:[-1, \infty) \longrightarrow X$ and $u_{t}:[-1,0] \longrightarrow X$ is defined by $u_{t}(\sigma):=u(t+\sigma)$. 
Definition 2.1. We say that a function $u:[-1, \infty) \longrightarrow X$ is a (classical) solution of $(D E)$ if

(i) $u \in C([-1, \infty), X) \cap C^{1}([0, \infty), X)$,

(ii) $u(t) \in D(B)$ and $u_{t} \in W^{1, p}([-1,0], X)$ for all $t \geq 0$, and

(iii) $u$ satisfies (DE) for all $t \geq 0$.

To be able to solve (DE) by semigroup methods, we introduce the Banach space

$$
\mathcal{E}:=X \times L^{p}([-1,0], X)
$$

with an arbitrary product norm, usually the $p$-norm, and the operator

$$
\mathcal{A}:=\left(\begin{array}{cc}
B & \Phi \\
0 & \frac{d}{d \sigma}
\end{array}\right)
$$

with domain

$$
D(\mathcal{A}):=\left\{\left(\begin{array}{l}
x \\
f
\end{array}\right) \in D(B) \times W^{1, p}([-1,0], X): f(0)=x\right\} .
$$

Consider now the abstract Cauchy problem

$$
(\mathrm{ACP}) \quad\left\{\begin{array}{l}
v^{\prime}(t)=\mathcal{A} v(t), \quad t \geq 0 \\
v(0)=v_{0}
\end{array}\right.
$$

associated to the operator matrix $(\mathcal{A}, D(\mathcal{A}))$ on the Banach space $\mathcal{E}$ with initial value $v_{0}:=\left(\begin{array}{l}x \\ f\end{array}\right)$. There is a natural correspondence between the solutions of the two problems (see [1, Proposition 2.3 and 2.4]).

Lemma 2.2. (i) If $u$ is a solution of $(D E)$, then $t \mapsto\left(\begin{array}{c}u(t) \\ u_{t}\end{array}\right)$ is a solution of the equation $(A C P)$.

(ii) If $t \mapsto\left(\begin{array}{c}u(t) \\ v(t)\end{array}\right)$ is a solution of $(A C P)$, then $v(t)=u_{t}$ for all $t \geq 0$ and $u$ is a solution of $(D E)$.

We can then give the following definition for well-posedness.

Definition 2.3. We say that (DE) is well-posed if

(i) for every $\left(\begin{array}{l}x \\ f\end{array}\right) \in D(\mathcal{A})$ there is a unique solution $u(x, f, \cdot)$, and

(ii) the solutions depend continuously on the initial values, i.e., if a sequence $\left(\begin{array}{l}x_{n} \\ f_{n}\end{array}\right)$ in $D(\mathcal{A})$ converges to $\left(\begin{array}{l}x \\ f\end{array}\right) \in D(\mathcal{A})$, then $u\left(x_{n}, f_{n}, t\right)$ converges to $u(x, f, t)$ uniformly for $t$ in compact intervals.

There is also a correspondence between the well-posedness of equation (DE) and of the abstract Cauchy problem (ACP), see also [1, Theorem 2.8].

Proposition 2.4. Let $(\mathcal{A}, D(\mathcal{A}))$ be the operator matrix defined by (2.1) and (2.2). Then the following assertions are equivalent. 
(i) Equation (DE) is well-posed.

(ii) $(\mathcal{A}, D(\mathcal{A}))$ is the generator of a strongly continuous semigroup on $\mathcal{E}$.

As a consequence of Lemma2.2 and Proposition 2.4, we have that if $(\mathcal{A}, D(\mathcal{A}))$ generates a strongly continuous semigroup $(\mathcal{T}(t))_{t \geq 0}$, then the solutions of equation (DE) are given by the first component of the function $t \mapsto \mathcal{T}(t)\left(\begin{array}{l}x \\ f\end{array}\right)$ for $\left(\begin{array}{l}x \\ f\end{array}\right) \in D(\mathcal{A})$.

By means of the perturbation theorem of Miyadera-Voigt (see [23, 30, 31] and [8, Corollary III.3.16]) one can formulate the following sufficient condition for the well-posedness of (DE), see [1, Theorem 3.3, Examples 3.4], [22].

Corollary 2.5. Assume that $(B, D(B))$ generates a strongly continuous semigroup $(S(t))_{t \geq 0}$ on $X, \infty>p \geq 1$, and that there exists a function $\eta:[-1,0] \rightarrow \mathcal{L}(X)$ of bounded variation such that $\Phi: C([-1,0], X) \rightarrow X$ is given by the Riemann-Stieltjes integral

$$
\Phi(f):=\int_{-1}^{0} d \eta f .
$$

Then $(\mathcal{A}, D(\mathcal{A}))$ is a generator on $\mathcal{E}$.

An important special case is the operator $\Phi$ defined by

$$
\Phi(f):=\sum_{k=0}^{n} B_{k} f\left(h_{k}\right), \quad f \in W^{1, p}([-1,0], X),
$$

where $B_{k} \in \mathcal{L}(X)$ and $h_{k} \in[-1,0]$ for $k=0, \ldots, n$.

It was also shown in [1] that the class of delay operators considered in Corollary 2.5 satisfies the following.

Definition 2.6. We call the delay operator $\Phi \in \mathcal{L}\left(W^{1, p}([-1,0], X), X\right)$ admissible if

(a) the operator $(\mathcal{A}, D(\mathcal{A}))$ is a generator for each generator $(B, D(B))$ and

(b) the function $\lambda \mapsto \Phi R\left(\lambda, A_{0}\right)$ is a bounded analytic function on the halfplane $\{\lambda \in$ $\mathbb{C}: \Re \lambda>\omega\}$ for all $\omega \in \mathbb{R}$.

We now characterize the resolvent set and the resolvent operator of $\mathcal{A}$ (see [1, Lemma

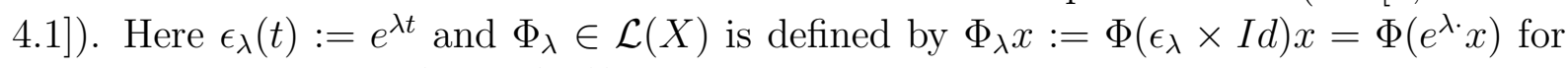
$x \in X$. The operator $\left(A_{0}, D\left(A_{0}\right)\right)$ is the generator of the nilpotent left shift semigroup $\left(T_{0}(t)\right)_{t \geq 0}$ in $L^{p}([-1,0], X)$.

Lemma 2.7. Let $X$ be a Banach space, $(B, D(B))$ be linear, closed and densely defined, and $\Phi: W^{1, p}([-1,0], X) \longrightarrow X$ be linear and bounded. Let $(\mathcal{A}, D(\mathcal{A}))$ be the operator matrix defined in (2.1) and (2.2). Then $\lambda \in \rho(\mathcal{A})$ if and only if $\lambda \in \rho\left(B+\Phi_{\lambda}\right)$. Moreover, for $\lambda \in \rho(\mathcal{A})$ the resolvent $R(\lambda, \mathcal{A})$ is given by

$$
\left(\begin{array}{cc}
R\left(\lambda, B+\Phi_{\lambda}\right) & R\left(\lambda, B+\Phi_{\lambda}\right) \Phi R\left(\lambda, A_{0}\right) \\
\epsilon_{\lambda} \otimes R\left(\lambda, B+\Phi_{\lambda}\right) & {\left[\epsilon_{\lambda} \otimes R\left(\lambda, B+\Phi_{\lambda}\right) \Phi+I d\right] R\left(\lambda, A_{0}\right)}
\end{array}\right)
$$




\section{$3 \quad$ Hyperbolicity and stability}

In the following, assume that $X$ is a Hilbert space and $p=2$. It follows that $\mathcal{E}$ will be also a Hilbert space and we may use the powerful Gearhart spectral mapping theorem (see e.g. [9, 17, 27]) to characterize hyperbolicity and exponential stability of the delay semigroup. The theorem can be found, e.g., in [8, Theorem V.1.11] and [8, Theorem V.1.18] in the form we quote it. For the hyperbolicity, this means that a semigroup $(T(t))_{t \geq 0}$ with generator $(G, D(G))$ in a Hilbert space $X$ is hyperbolic if and only if $i \mathbb{R} \subset \rho(G)$ and $\sup \{\|R(i \omega, G)\|: \omega \in \mathbb{R}\}<\infty$. There are recent generalizations of this result to the Banach space case, see [18, 21], which may allow in the near future to generalize the results presented here to the Banach space case.

Theorem 3.1. Let $X$ be a Hilbert space and consider the equation (DE). Assume that $\Phi$ is admissible, the semigroup $(B, D(B))$ generates a hyperbolic semigroup and consider

$$
a_{n}:=\sup _{\omega \in \mathbb{R}}\left\|\left(\Phi_{i \omega} R(i \omega, B)\right)^{n}\right\|<\infty .
$$

If

$$
a:=\sum_{n=0}^{\infty} a_{n}<\infty
$$

then $(\mathcal{A}, D(\mathcal{A}))$ generates a hyperbolic semigroup.

Proof. As a consequence of the above mentioned Theorem of Gearhart, the numbers $a_{n}$ are defined for all $n \in \mathbb{N}$ and we have to show the boundedness of the resolvent operator given in (2.4) on the line $i \mathbb{R}$. Under our assumptions, this is equivalent to the existence and boundedness of $R\left(\lambda, B+\Phi_{\lambda}\right)$ on the line $\{\lambda \in i \mathbb{R}\}$.

Defining $M:=\sup _{\lambda \in i \mathbb{R}}\|R(\lambda, B)\|$, we obtain for all $\lambda \in i \mathbb{R}$ that

$$
R(\lambda, B) \sum_{n=0}^{\infty}\left(\Phi_{\lambda} R(\lambda, B)\right)^{n} \in \mathcal{L}(X)
$$

and

$$
\left\|R(\lambda, B) \sum_{n=0}^{\infty}\left(\Phi_{\lambda} R(\lambda, B)\right)^{n}\right\| \leq M \sum_{n=0}^{\infty}\left\|\left(\Phi_{\lambda} R(\lambda, B)\right)^{n}\right\| \leq M \sum_{n=0}^{\infty} a_{n}=M \cdot a .
$$

Easy calculations show that this operator defines an inverse for $\left(\lambda-B-\Phi_{\lambda}\right)$ being bounded on the line $i \mathbb{R}$.

Corollary 3.2. Assume that $X$ is a Hilbert space, $\Phi$ is admissible, and $(B, D(B))$ generates a hyperbolic semigroup. If

$$
\sup _{\omega \in \mathbb{R}}\left\|\Phi_{i \omega} R(i \omega, B)\right\|<1
$$

or in particular if

$$
\sup _{\omega \in \mathbb{R}}\left\|\Phi_{i \omega}\right\|<\frac{1}{\sup _{\omega \in \mathbb{R}}\|R(i \omega, B)\|},
$$

then $(\mathcal{A}, D(\mathcal{A}))$ generates a hyperbolic semigroup. 
The proof is an easy consequence of the previous theorem using Weierstrass' criterion on the convergence of infinite series.

As an important special case of hyperbolicity, we may now consider uniform exponential stability.

Theorem 3.3. Let $X$ be a Hilbert space and consider the equation (DE). Assume that $\Phi$ is admissible, that $\omega_{0}(B)<0$, and consider

$$
a_{n}:=\sup _{\omega \in \mathbb{R}}\left\|\left(\Phi_{i \omega} R(i \omega, B)\right)^{n}\right\|<\infty .
$$

If

$$
a:=\sum_{n=0}^{\infty} a_{n}<\infty
$$

then $\omega_{0}(\mathcal{A})<0$.

Proof. It follows from the inequality

$$
\sup _{\omega \in \mathbb{R}}\left\|\left(\Phi_{\alpha+i \omega} R(\alpha+i \omega, B)\right)^{n}\right\| \leq \sup _{\omega \in \mathbb{R}}\left\|\left(\Phi_{i \omega} R(i \omega, B)\right)^{n}\right\|
$$

for all $\alpha \geq 0$, which is a consequence of the generalized maximum principle, and from Theorem 3.1 that the semigroup generated by $(\mathcal{A}-\alpha)$ is hyperbolic for all $\alpha \geq 0$. Thus, $\omega_{0}(\mathcal{A})<0$.

Corollary 3.4. Assume that $X$ is a Hilbert space, $\Phi$ is admissible, $\omega_{0}(B)<0$ and let $\alpha \in\left(\omega_{0}(B), 0\right]$. If

$$
\sup _{\omega \in \mathbb{R}}\left\|\Phi_{\alpha+i \omega} R(\alpha+i \omega, B)\right\|<1
$$

or in particular if

$$
\sup _{\omega \in \mathbb{R}}\left\|\Phi_{\alpha+i \omega}\right\|<\frac{1}{\sup _{\omega \in \mathbb{R}}\|R(\alpha+i \omega, B)\|},
$$

then $\omega_{0}(\mathcal{A})<\alpha \leq 0$.

We demonstrate in the following example that the results obtained in Theorem 3.3 are more general then the ones in 1 .

Corollary 3.5. Assume that $X$ is a Hilbert space, $\Phi=C \delta_{-1}$ for $C \in \mathcal{L}(X)$ commuting with $(B, D(B))$, that $\omega_{0}(B)<0$ and that

$$
r(C)<\frac{1}{\sup _{\omega \in \mathbb{R}}\|R(i \omega, B)\|} .
$$

Then $\omega_{0}(\mathcal{A})<0$.

Proof. We use ideas analogous to [20, Theorem IV.3.6]. Let us denote by $M:=$ $\sup _{\omega \in \mathbb{R}}\|R(i \omega, B)\|$. Our assumption means that there exists $0<q<1$ such that $r(C) \cdot M<q<1$. We obtain that there exists $n_{0} \in \mathbb{N}$ such that

$$
\left\|C^{n}\right\|^{\frac{1}{n}} \cdot M<q<1 \quad \text { for all } n \geq n_{0} .
$$

This means that

$$
a_{0, n}=\sup _{\omega \in \mathbb{R}}\left\|\left(\Phi_{i \omega} R(i \omega, B)\right)^{n}\right\| \leq \sup _{\omega \in \mathbb{R}}\left\|R(i \omega, B)^{n}\right\| \sup _{\omega \in \mathbb{R}}\left\|\Phi_{i \omega}^{n}\right\| \leq\left\|C^{n}\right\| \cdot M^{n}<q^{n}
$$

for $n \geq n_{0}$, and the assertion follows by Weierstrass' criterion. 


\section{An application: the effect of small delays}

The problem considered in this section is the following: Assume that hyperbolicity or uniform exponential stability is known for the solutions of the equation

$$
(\mathrm{DE})_{0} \quad\left\{\begin{array}{l}
u^{\prime}(t)=(B+C) u(t), \quad t \geq 0 \\
u(0)=x \\
u_{0}=f
\end{array}\right.
$$

where $C \in \mathcal{L}(X)$.

The question is, whether the same type of asymptotics holds for the solutions of the equation

$$
(\mathrm{DE})_{\tau} \quad\left\{\begin{array}{l}
u^{\prime}(t)=B u(t)+C u(t-\tau), \quad t \geq 0 \\
u(0)=x \\
u_{0}=f
\end{array}\right.
$$

where $\tau>0$ is "small".

It is known, see e.g. [25, Example B-IV.3.10], that if $X$ is a Banach lattice, $C$ is a positive operator and $(B, D(B))$ generates a positive semigroup, then the solutions of $(\mathrm{DE})_{\tau}$ are uniformly exponentially stable if and only if the solutions of $(\mathrm{DE})_{0}$ are uniformly exponentially stable.

It is an open question however, what happens to the hyperbolicity in the positive case. The example of Montgomery-Smith [24] suggests that this result may not remain true.

The first who examined this effect was R. Datko [3, 4, 5, 6, 7]. It is known for finite dimensional equations that the stability cannot be destroyed and there exists an extensive literature on delay dependent stability conditions, see e.g. [11, 12]. For similar questions in the parabolic case we refer to [10, 29]. There is a recent exposition of this problem by J. Hale and S. Verduyn Lunel [15, 16], where many examples of functional differential and difference equations are considered. A control theoretical investigation using transfer functions was made for compact feedback in [28].

Before considering the abstract problem, we demonstrate on some simple examples how the stability can be destroyed. Though the following example seems to be known, we include it here because it is the simplest example and we could not find it written in the literature.

Example 4.1. Let $(B, D(B))$ be the (unbounded) generator of a unitary group in an infinite dimensional Hilbert space $H$ and let $C:=d \cdot I d$ for $d<0$. Then $(B+C, D(B))$ generates an exponentially stable semigroup. We show that there exists a sequence $\left(\tau_{k}\right)$, $\tau_{k} \rightarrow 0$, such that the solution semigroup of the equation $(\mathrm{DE})_{\tau_{k}}$ does not decay exponentially for each $k \in \mathbb{N}$.

To construct this sequence, take $\left(\mu_{k}\right) \subset \mathbb{R}, i \mu_{k} \in \sigma(B)$ such that $\left|\mu_{k}\right| \rightarrow \infty$ and $\mu_{k} \neq-d$. Defining the numbers

$$
\tau_{k}:= \begin{cases}\frac{3 \pi}{2\left(\mu_{k}+d\right)}, & \mu_{k}+d>0 \\ \frac{-\pi}{2\left(\mu_{k}+d\right)}, & \mu_{k}+d<0\end{cases}
$$


and the operators $\Phi^{(k)}:=d \cdot I d \delta_{-\tau_{k}}, \Phi_{\lambda}^{(k)}:=e^{-\lambda \tau_{k}} d \cdot I d$, we obtain for the numbers $\lambda_{k}:=\left(\mu_{k}+d\right) i \in i \mathbb{R}$ that

$$
\lambda_{k} \in \sigma\left(B+\Phi^{(k)} i_{\lambda_{k}}\right)=\sigma(B)+d \cdot e^{-\lambda_{k} \tau_{k}}=\sigma(B)+d \cdot i
$$

By the spectral characterization in Lemma 2.7 it follows that the associated operator $(\mathcal{A}, D(\mathcal{A}))$ can not generate a uniformly exponentially stable semigroup. If we assume further that $i \mu_{k} \in P \sigma(B)$, which e.g., is satisfied if $(B, D(B))$ has compact resolvent, then we also find classical solutions of $(\mathrm{DE})_{\tau_{k}}$ which are not decaying exponentially.

The essence of this example can be formulated as follows.

Theorem 4.2. Let $X$ be a Hilbert space and assume that $(B, D(B))$ generates a strongly continuous semigroup such that there exists $\rho \in \mathbb{R}$ and $\mu_{k} \in \mathbb{R},\left|\mu_{k}\right| \rightarrow \infty$ such that $\rho+i \mu_{k} \in \sigma(B)$, i.e., the spectrum is unbounded along an imaginary line. Then there exists $C \in \mathcal{L}(X)$ and $\left(\tau_{k}\right) \subset \mathbb{R}^{+}, \tau_{k} \rightarrow 0$ such that $\omega_{0}(B+C)<0$ but the solutions of $(\mathrm{DE})_{\tau}$ are not uniformly exponentially stable.

Proof. As in the previous example, take $C:=-\mu \cdot I d, \mu>0, \mu>-\rho$ and define

$$
\tau_{k}:=\frac{\pi}{\left|\mu_{k}\right|}
$$

for some $k \in \mathbb{N}$. Then the corresponding characteristic equations are again

$$
z-\lambda+\mu e^{-z \tau_{k}}=0, \quad \lambda \in \sigma(B) .
$$

Now take $\lambda=\rho+i \mu_{k}$ and put $z=\varepsilon+i \mu_{k}$ for some $\varepsilon>0$. Then we obtain

$$
i \mu_{k}+\varepsilon-\rho-i \mu_{k}+\mu e^{-i \mu_{k} \tau_{k}} \cdot e^{-\varepsilon \tau_{k}}=0
$$

and hence

$$
\varepsilon=\mu e^{-\varepsilon \tau_{k}}+\rho .
$$

Since $\mu>-\rho$, there exists a positive real solution $\varepsilon$.

Unfortunately, if the stabilizing operator is not the identity, the preceding technique can be applied only with enormous difficulty even in cases where the spectral mapping theorem holds. This is because we have in general no easy characterization of $\sigma\left(B+\Phi_{\lambda}\right)$, see [20, Example IV. 3.8].

Turning our attention now to the general problem of small delays, we use an idea similar to [14, Section $5.4(4.9)$ ] and transform the equation $(\mathrm{DE})_{\tau}$ into

$$
u^{\prime}(t)=(B+C) u(t)+C(u(t-\tau)-u(t)) .
$$

We use the equality

$$
u\left(t_{2}\right)-u\left(t_{1}\right)=\left[S\left(t_{2}-t_{1}\right)-I d\right] u\left(t_{1}\right)+\int_{t_{1}}^{t_{2}} S\left(t_{2}-s\right) C u(s-\tau) d s
$$


for $t_{2}>t_{1} \geq 0$ following from $(\mathrm{DE})_{\tau}$. Substituting $t_{1}=t-\tau$ and $t_{2}=t$, we obtain that

$$
u(t)-u(t-\tau)=[S(\tau)-I d] u(t-\tau)+\int_{-\tau}^{0} S(-s) C u(t+s-\tau) d s .
$$

Thus, $(\mathrm{DE})_{\tau}$ can be written in the form

$$
\begin{aligned}
u^{\prime}(t)=(B+C) u(t) & \\
& -C\left([S(\tau)-I d] u(t-\tau)+\int_{-\tau}^{0} S(-s) C u(t+s-\tau) d s\right) .
\end{aligned}
$$

Defining

$$
\Phi f:=-C[S(\tau)-I d] \delta_{-\tau} f-\int_{-\tau}^{0} C S(-s) C \delta_{s-\tau} f d s,
$$

where $\delta_{r} \in \mathcal{L}\left(W^{1, p}([-1,0], X), X\right)$ is given by $\delta_{r}(f):=f(r)$ for $r \in[-1,0]$, we see that the previous stability results in Corollary 3.4 are applicable to our original problem and that $(\mathrm{DE})_{\tau}$ has the form

$$
u^{\prime}(t)=(B+C) u(t)+\Phi u_{t}
$$

In order to be able to apply the stability results of Corollary $\underline{3.4}$, or the hyperbolicity results of Corollary [3.2, we have to calculate

$$
\begin{aligned}
\Phi_{\lambda} R(\lambda, B+C) x=-C[S(\tau)-I d] e^{-\lambda \tau} & R(\lambda, B+C) x \\
& -\int_{-\tau}^{0} C S(-s) C e^{-\lambda(s-\tau)} R(\lambda, B+C) x d s .
\end{aligned}
$$

Defining

$$
I_{1}^{\omega}(\tau):=C[S(\tau)-I d] e^{-i \omega \tau} R(i \omega, B+C)
$$

and

$$
I_{2}^{\omega}(\tau) x:=\int_{-\tau}^{0} C S(-s) C e^{-i \omega(s-\tau)} R(i \omega, B+C) x d s
$$

it would be sufficient to show that there exists $\kappa>0$ such that $\sup _{\omega \in \mathbb{R}}\left\|I_{i}^{\omega}(\tau)\right\|<\frac{1}{2}$ for $i=1,2$ and all $\tau \in(0, \kappa)$. Then, using Corollary 3.4 or Corollary 3.2 and (4.5), the assertion follows since $\sup _{\omega \in \mathbb{R}}\left\|\Phi_{i \omega} R(i \omega, B+C)\right\| \leq \sup _{\omega \in \mathbb{R}}\left\|I_{1}^{\omega}(\tau)\right\|+\sup _{\omega \in \mathbb{R}}\left\|I_{2}^{\omega}(\tau)\right\|<1$.

The estimate on $I_{2}^{\omega}$ is

$$
\left\|I_{2}^{\omega}(\tau)\right\| \leq \tau\|C\|^{2} K\|R(i \omega, B+C)\|
$$

where $K:=\sup _{0 \leq t \leq 1}\|S(t)\|$. Since $\|R(i \omega, B+C)\|$ is uniformly bounded for all $\omega \in \mathbb{R}$, there exists $\kappa_{2}>0$ such that for all $\tau \in\left(0, \kappa_{2}\right)$ the estimate $\sup _{\omega \in \mathbb{R}}\left\|I_{2}^{\omega}(\tau)\right\|<\frac{1}{2}$ holds.

The estimate on $I_{1}^{\omega}$ is

$$
\begin{aligned}
\left\|I_{1}^{\omega}(\tau)\right\| \leq\|C\| \cdot \|(S(\tau) & -I d) R(i \omega, B+C) \| \\
& \leq\|C\| \cdot\|(S(\tau)-I d) R(\lambda, B)\| \cdot\|(\lambda-B) R(i \omega, B+C)\|,
\end{aligned}
$$


where $\lambda>\max \left\{\omega_{0}(B), 0\right\}$ is fixed.

Since $\|(\lambda-B) R(i \omega, B+C)\|$ is independent of $\tau$, we only have to consider the term $(S(\tau)-I d) R(\lambda, B)$.

But then it follows from

$$
\begin{aligned}
\|(S(\tau)-I d) R(\lambda, B)\| \leq\|S(\tau)\|\left(1-e^{-\lambda \tau}\right)\|R(\lambda, B)\|+\left\|\int_{0}^{\tau} e^{-\lambda s} S(s) d s\right\| & \\
& \leq \tau K(\|R(\lambda, B)\||\lambda|+1)
\end{aligned}
$$

that for every $\omega \in \mathbb{R}$

$$
\lim _{\tau \rightarrow 0}\left\|C[S(\tau)-I d] e^{i \omega \tau} R(i \omega, B+C)\right\|=0
$$

Example 4.1 shows that in general this convergence cannot be uniform. This is the point where we need some extra assumptions.

As we could see in Example 4.1, the unboundedness of the spectrum of $(B, D(B))$ along imaginary axes may cause trouble if we allow any stabilizing operator $C$. In the following result on the independence of stability of small delays the spectrum of the generator also plays an important role.

Theorem 4.3. Assume that $(B, D(B))$ generates an immediately norm continuous semigroup and that the semigroup generated by $(B+C, D(B))$ is exponentially stable or hyperbolic in the Banach space $X$. Then there exists $\kappa>0$ such that the solution semigroup of $(\mathrm{DE})_{\tau}$ is exponentially stable or hyperbolic, respectively, for all $\tau \in(0, \kappa)$. Thus, the stability and the hyperbolicity is not sensitive to small delays.

Proof. We have to show that the convergence in (4.10) is uniform in $\omega$.

To this end we use the immediate norm continuity of the semigroup generated by $(B+C, D(B))$, see [8, Theorem III.1.16(i)]. An important consequence is that $\lim _{|\omega| \rightarrow \infty}\|R(i \omega, B+C)\|=0$, see [8, Corollary II.4.19]. Thus, there exists $L>0$ such that

$$
\|R(i \omega, B+C)\|<\frac{1}{2\|C\|(K+1)} \text { for }|\omega|>L,
$$

where $K:=\sup _{0 \leq t \leq 1}\|S(t)\|$.

For $\omega \in[-L, L]$, we recall that the function

$$
(\omega, \tau) \mapsto\left\|C[S(\tau)-I d] e^{i \omega \tau} R(i \omega, B+C)\right\|
$$

is uniformly continuous on $[-L, L] \times[0,1]$. Thus, there exists $\kappa_{1}>0$ such that for all $\tau \in\left(0, \kappa_{1}\right)$ and for all $\omega \in[-L, L]$

$$
\left\|C[S(\tau)-I d] e^{i \omega \tau} R(i \omega, B+C)\right\|<\frac{1}{2} .
$$

Combining these estimates we obtain the desired statement.

The proof can be finished by choosing $\kappa:=\min \left\{\kappa_{1}, \kappa_{2}\right\}$. 
We make the remark that the results of the previous theorem remain true also if $X$ is a Banach space. This is because under the conditions of the theorem, the delay semigroup will be eventually norm continuous, see [1, Proposition 5.3], and for eventually norm continuous semigroups the spectral mapping theorem holds, see [8, Theorem IV.3.10].

Analogous results were obtained by R. Schnaubelt [29] for non-autonomous equations in the parabolic case.

In the previous theorem we gave a condition on the generator $(B, D(B))$ without any restriction on the stabilizing operator $C$. In the following we also provide a condition involving $C$.

Proposition 4.4. Let $(B, D(B))$ be a generator of a strongly continuous semigroup $(S(t))_{t \geq 0}$ in the Hilbert space $X, C \in \mathcal{L}(X)$ be a compact operator commuting with $B$ and that the semigroup generated by $(B+C, D(B))$ is exponentially stable or hyperbolic in the Hilbert space $X$. Then there exists $\kappa>0$ such that the solution semigroup of $(\mathrm{DE})_{\tau}$ is exponentially stable or hyperbolic for all $\tau \in(0, \kappa)$. Thus, the stability and the hyperbolicity is not sensitive to small delays.

Proof. Again we only have to show that the convergence in (4.10) is uniform in $\omega$. Using that $C$ commutes with $B$ and hence with the semigroup, we obtain

$$
C[S(\tau)-I d] e^{i \omega \tau} R(i \omega, B+C)=[S(\tau)-I d] e^{i \omega \tau} R(i \omega, B+C) C .
$$

By our assumptions, the set $C B(0,1) \subset X$ is precompact in $X$. The proof can be finished by using the fact that on compact sets the strong and the uniform topology coincide.

Remark 4.5. To show that estimating $I_{1}^{\omega}$ and $I_{2}^{\omega}$ is not sharp, consider the well-known example $B=0$ and $C=d \cdot I d$ for $d<0$ in the Banach space $\mathbb{C}$. The direct calculation of the formula (4.5) shows that the solutions of $(\mathrm{DE})_{\tau}$ are exponentially stable if $|d| \tau<1$.

However, applying directly the spectral characterization of Lemma 2.7 and using that $\sigma\left(B+\Phi_{\lambda}\right)=\left\{d \cdot e^{-\lambda \tau}\right\}$, we obtain the well-known and best possible estimate (see [14, p. 135]) that the solutions decay exponentially if

$$
|d| \tau<\frac{\pi}{2}
$$

\section{References}

[1] Bátkai, A., Piazzera, S., Semigroups and linear partial differential equations with delay, to appear in J. Math. Anal. Appl.

[2] Bátkai, A., Piazzera, S., Damped wave equations with delay, Fields Institute Communications 29 (2001), 51-61.

[3] Datko, R., Is boundary control a realistic approach to the stabilization of vibrating elastic systems?, in: Ferreyra, Guillermo (eds.), "Evolution Equations", Marcel Dekker, 133-140 (1994).

[4] Datko, R. Two questions concerning the boundary control of elastic systems, J. Diff. Eq. 92 (1991), 27-44. 
[5] Datko, R. Not all feedback stabilized systems are robust with respect to small time delays in their feedback, SIAM J. Control and Optimization 26 (1988), 697-713.

[6] Datko, R., Lagnese, J., Polis, M. P., An example on the effect of time delays in boundary feedback of wave equations, SIAM J. Control and Optimization 24 (1986), $152-156$.

[7] Datko, R., You, Y.C., Some second order vibrating systems cannot tolerate small time delays in their damping, J. Optim. Th. Appl. 70 (1991), 521-537.

[8] Engel, K.-J., Nagel R., "One-parameter Semigroups for Linear Evolution Equations", Graduate Texts in Mathematics 194, Springer-Verlag, 1999.

[9] Gearhart, L., Spectral theory for contraction semigroups on Hilbert space, Trans. Amer. Math. Soc. 236 (1978), 385-394.

[10] Gühring, G., Räbiger, F., Schnaubelt, R., A characteristic equation for nonautonomous partial functional differential equations, preprint, 2000.

[11] Győri, I., Pituk, M., Stability criteria for linear delay differential equations, Diff. Int. Eq. 10 (1997), 841-852.

[12] Győri, I., Hartung, F., Turi, J., Preservation of stability in delay equations under delay perturbations, J. Math. Anal. Appl. 220 (1998), 290-313.

[13] Hale, J. K., "Functional Differential Equations", Appl. Math. Sci., vol. 3, SpringerVerlag, 1971.

[14] Hale, J. K., Verduyn Lunel, S. M., "Introduction to Functional Differential Equations", Appl. Math. Sci. 99, Springer-Verlag, 1993.

[15] Hale, J. K., Verduyn Lunel, S. M., Effects of small delays on stability and control, in: Bart, Gohberg, Ran (eds), "Operator Theory and Analysis, The M. A. Kaashoek Anniversary Volume", Operator Theory: Advances and Applications, Vol. 122, Birkhäuser, 275-301 (2001).

[16] Hale, J. K., Verduyn Lunel, S. M., Effects of time delays on the dynamics of feedback systems, in: Fiedler, Gröger, Sprekels (eds.), "EQUADIFF'99, International Conference on Differential Equations, Berlin 1999", World Scientific, 257-266 (2000).

[17] Herbst, I. W., The spectrum of Hilbert space semigroups, J. Op. Th. 10 (1983), 87-94.

[18] Kaashoek, M. A., Verduyn Lunel, S. M., An integrability condition on the resolvent for hyperbolicity of the semigroup, J. Diff. Eq. 112 (1994), 374-406.

[19] Kappel, F., Semigroups and delay equations, in: Brezis, H., Crandall, M. G., Kappel, F. (ed.), "Semigroups, Theory and Applications", Vol. II., Pitman Research Notes in Mathematics 152, Longman, 136-176 (1986).

[20] Kato, T., "Perturbation Theory for Linear Operators", Grundlehren Math. Wiss. 132, Springer-Verlag, 1980.

[21] Latushkin, Y., Shvydkoy, R., Hyperbolicity of semigroups and fourier multipliers, preprint, 2000.

[22] Maniar, L., Voigt, J., Linear delay equations in the $L^{p}$ context, Preprint, 2000.

[23] Miyadera, I., On perturbation theory for semi-groups of operators, Tôhoku Math. 18, 299-310 (1966). 
[24] Montgomery-Smith, S., Stability and dichotomy of positive semigroups in $L^{p}$, Proc. Am. Math. Soc. 124 (1996), 2433-2437.

[25] Nagel, R. (ed.), "One-parameter Semigroups of Positive Operators", Springer-Verlag, 1986.

[26] Nakagiri, S., Optimal control of linear retarded systems in Banach spaces, J. Math. Anal. Appl. 120 (1986), 169-210.

[27] Prüß, J., On the spectrum of $C_{0}$-semigroups, Trans. Amer. Math. Soc. 284 (1984), $847-857$.

[28] Rebarber, R., Townly, S., Robustness with respect to delays for exponential stability of distributed parameter systems, SIAM J. Control and Optimization 37 (1998), 230244.

[29] Schnaubelt, R., Parabolic evolution equations with asymptotically autonomous delay, preprint, 2001.

[30] Voigt, J., On the perturbation theory for strongly continuous semigroups, Math. Ann. 229, 163-171 (1977).

[31] Voigt, J., Absorption semigroups, Feller property, and Kato class, Oper. Theory. Adv. Appl. 78, 389-396 (1995).

[32] Webb, G., Functional differential equations and nonlinear semigroups in $L^{p}$-spaces, J. Diff. Eq. 29 (1976), 71-89.

[33] Wu, J., "Theory and Applications of Partial Functional Differential Equations", Appl. Math. Sci. 119, Springer-Verlag, 1996.

ELTE TTK, Department of Applied Analysis, Pf. 120, H-1518 Budapest, Hungary e-mail: batka@cs.elte.hu

AMS Classification Numbers: 34K05, 34K20, 47D06 